\title{
La poussée radiale dans les machines hydrauliques: expériences de laboratoire
}

\author{
Radial thrust in hydraulic machines: laboratory testing
}

\author{
V. Zanetti \\ Vice-Directeur du Développement et de la Recherche, \\ Hydroart S.p.A. Milan
}

\section{Introduction}

Aujourd'hui particulièrement on sent l'importance de la connaissance des valeurs que peut prendre la poussée radiale sur la roue d'une machine hydraulique dans les différentes conditions de fonctionnement.

En effet, la poussée radiale est devenue un paramètre très important pour le dimensionnement de la ligne d'axe et des paliers, à cause des dimensions de plus en plus grandes et des performances de plus en plus élevées des machines hydrauliques pour centrales hydroélectriques. La nécessité d'évaluer la grandeur des poussées, concerne non seulement le fonctionnement dans les conditions normales, mais aussi et surtout les fonctionnements aux charges partielles et à l'emballement ainsi que - sous un autre aspect - ceux en période transitoire (en particulier le passage d'une réversible du fonctionnement en pompe à celui en turbine).

D'autre part, les difficultés remarquables que la mesure de la poussée radiale ont présenté autrefois sont aujourd'hui graduellement surmontées par l'emploi, de plus en plus étendu, des transducteurs de différents types et d'appareillages électroniques pour le traitement des signaux.

C'est surtout en laboratoire qu'il est possible de recueillir systématiquement les données nécessaires pour une étude poussée du phénomène, grâce à la possibilité de réaliser les conditions de fonctionnement les plus diverses et d'analyser les effets des modifications apportées à l'hydraulique de la machine (voir en appendice la description de l'instrumentation utilisée).

A cet égard, il faut remarquer aussi que les récents cahiers des charges des machines hydrauliques pour aménagements hydroélectriques prescrivent de plus en plus fréquemment de vérifier sur modèle les données de poussée radiale.

\section{Classification des poussées radiales}

Avant de passer à la présentation des résultats d'essai, il nous semble opportun de faire une tentative de classification des divers types de poussée qui peuvent se vérifier dans les turbines Francis ou les pompe-turbines, en mettant en évidence pour chacun d'eux le problème technique spécifique.

La classification ne peut qu'être schématique et il faut tenir compte, qu'en réalité, les divers types de poussée très souvent se superposent.

\section{a) Poussée radiale fixe (statique)}

Elle est caractérisée par une force fixe par rapport au stator, à direction et amplitude déterminées. En général, ces paramètres varient dans certaines limites selon les conditions de fonctionnement. La poussée radiale prend naissance par suite de la dissymétrie du stator par rapport à son axe et peut aussi bien avoir une cause organique (bâche spirale, coude d'aspiration) qu'accidentelle (par exemple asynchronisation d'une directrice, excentricité des labyrinthes du stator). Son effet a pour conséquence de charger latéralement les paliers guides et de fléchir la ligne d'axe, mais pas de la faire vibrer. Elle intéresse donc surtout le dimensionnement des paliers et du système de refroidissement relatif.

Dans certaines machines - pompes à hauteur élevée avec diffuseur sans aubage - son amplitude peut être telle qu'elle oblige à adopter une spirale à double volute pour rétablir la symétrie axiale, au moins en partie.

Au contraire, dans le cas des machines pour aménagements hydroélectriques, cette poussée ne pose pas de problème en raison de la présence systématique des aubes du distributeur ou du diffuseur, sauf dans le cas d'une asynchronisation accidentelle $\mathrm{du}$ distributeur. 


\section{b) Poussées radiales non-fixes (dynamiques)}

Ellès peuvent.. être divisées, à leur tour, en deux catégories principales :

b.1) celles caractérisées par une force à amplitude constante qui toume à une vitesse déterminée et

b.2) celles représentées par une force qui varie à chaque instant, soit en grandeur, soit en direction.

Il convient de séparer les premières en deux souscatégories :

- b.1.1. Poussée radiale tournante à la vitesse de la machine

Elle dérive des dissymètries de la partie tournante. Elle est pratiquement toujours présente mais en mesure généralement modeste.

- b.1.2. Poussée radiale tournante à la fréquence de la torche

Elle est caractéristique du fonctionnement à débit partiel d'une turbine Francis et prend des valeurs considérables dans les machines Francis à haute vitesse et à l'emballement. Dans ce dernier cas, il est nécessaire de bien la connaitre afin d'un dimensionnement correct de la machine et en particulier pour s'assurer que le déplacement radial de la roue, produit par la poussée, n'atteigne pas des valeurs telles qu'elles puissent causer le contact entre la partie tournante et la partie fixe.

\section{b.2. Poussée radiale aléatoire}

Elle se présente pratiquement dans tous les fonctionnements loin des conditions normales et est évidemment générée par les détachements de veine, les tourbillons etc. Elle comporte des problèmes de structure de la machine surtout pour la résistance à la fatigue. Vu l'intérêt technique des deux types de poussée b.1.2.) et b.2.) et compte tenu de l'argument traité par le groupe de travail, c'est à ces derniers que l'on a dédié le chapitre suivant qui résume les résultats des mesures sur modèle.

\section{Résultats des essais sur modèle}

\section{Poussée radiale tournante à la fréquence de la torche}

Dans une turbine Francis fonctionnant à débit partiel, le phénomène caractéristique d'instabilité dit "de torche" se vérifie quand le débit est inférieur à environ $50 \%$ de celui de pleine charge.

L'aspect toujours présent de ce phénomène, au moins dans les Francis à vitesse moyenne ou élevée, est justement la torche, en forme de spirale - visible au stroboscope quand la pression descend au-dessous de certaines valeurs. Cette torche qui tourne à une fréquence égale au tiers ou à quart de celle de la machine, est liée à la rotation synchrone du champ de pression en aval de la roue, et est plus ou moins camouflée par des pulsations du niveau général de pression.

Ce phénomène peut être accompagné par les pulsations susnommées, mais aussi par des pulsations analogues de la pression en amont, de la chute, du débit, du couple à l'arbre, de la poussée axiale, etc. : il est toujours accompagné par une poussée radiale du type b.1.2.
Le diagramme de figure 1, concernant le modèle d'une turbine Francis à haute vitesse caractéristique, montre - pour trois chutes de fonctionnement - les valeurs de la poussée radiale tournant à la fréquence de la torche (ligne épaisse). Le même diagramme indique aussi (ligne à traits) les pulsations de pression relevées immédiatement au dessous de la roue à l'intrados du coude. Toutes les grandeurs sont exprimées en fonction de l'ouverture du distributeur.
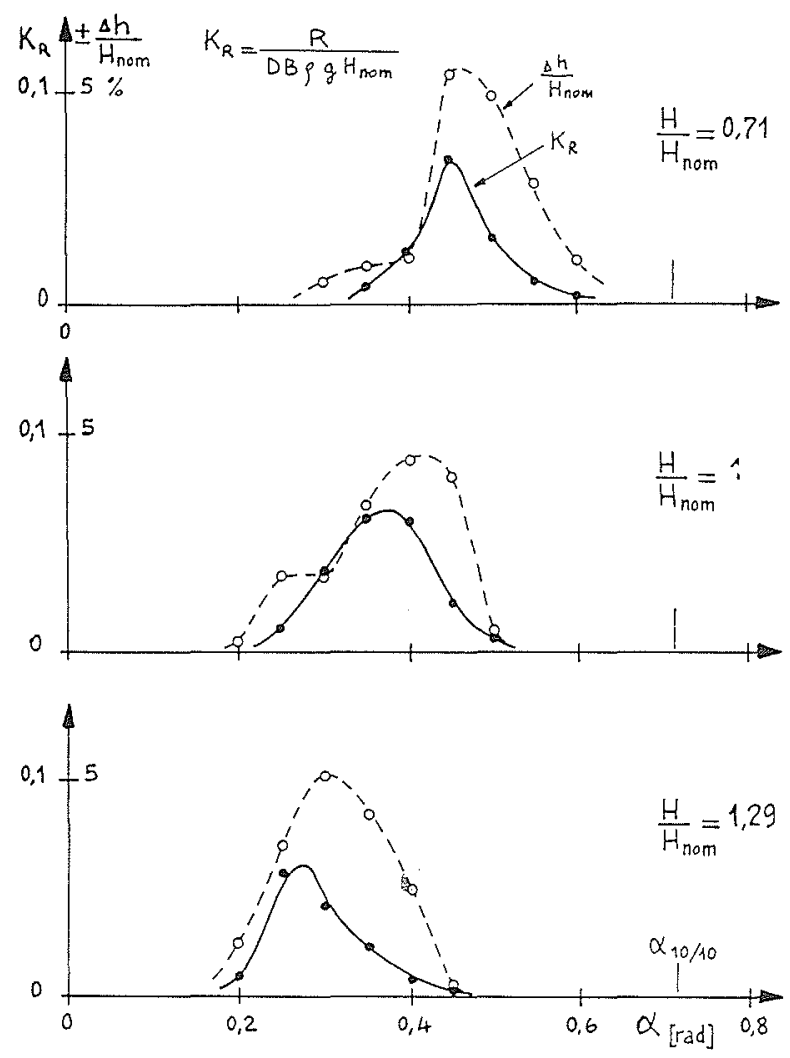

Figure 1 - Turbine Francis $n_{q_{-}}=95:$ poussée radiale de torche et pulsation de pression sous roue en fonction de l'ouverture du distributeur.

L'allure des courbes met clairement en évidence la corrélation entre les deux grandeurs. Le diagramme de la figure 2 montre les mêmes coefficients de poussée, exprimés cette fois en fonction du débit relatif : on remarquera que ce phénomène atteint l'amplitude maximale pour un certain débit, plus ou moins indépendant de la chute. Ce débit correspond à la moitié du débit théorique auquel cas, les triangles de sorties sont rectangles. Le diagramme de la figure 3 indique le coefficient de poussée radiale maximale de torche mesuré sur différents modèles de turbine Francis et de pompe-turbines monoétage en fonction de la vitesse spécifique.

La grandeur des poussées radiales, dans le fonctionnement dont on vient de parler, n'est toutefois pas telle qu'elle puisse créer des problèmes techniques, car les pulsations de charge sur les paliers et les déformations engendrées de la ligne d'axe ne demandent pas un dimensionnement spécial. Par contre, les poussées radiales qui peuvent se vérifier dans le phénomène 
analogue (eau à la sortie de la roue animée par une forte composante tangentielle) lors de l'emballement, sont sensiblement supérieures, surtout dans les machines à haute vitesse spécifique et par conséquent elles conditionnent, comme déjà mentionné, le projet de la structure.
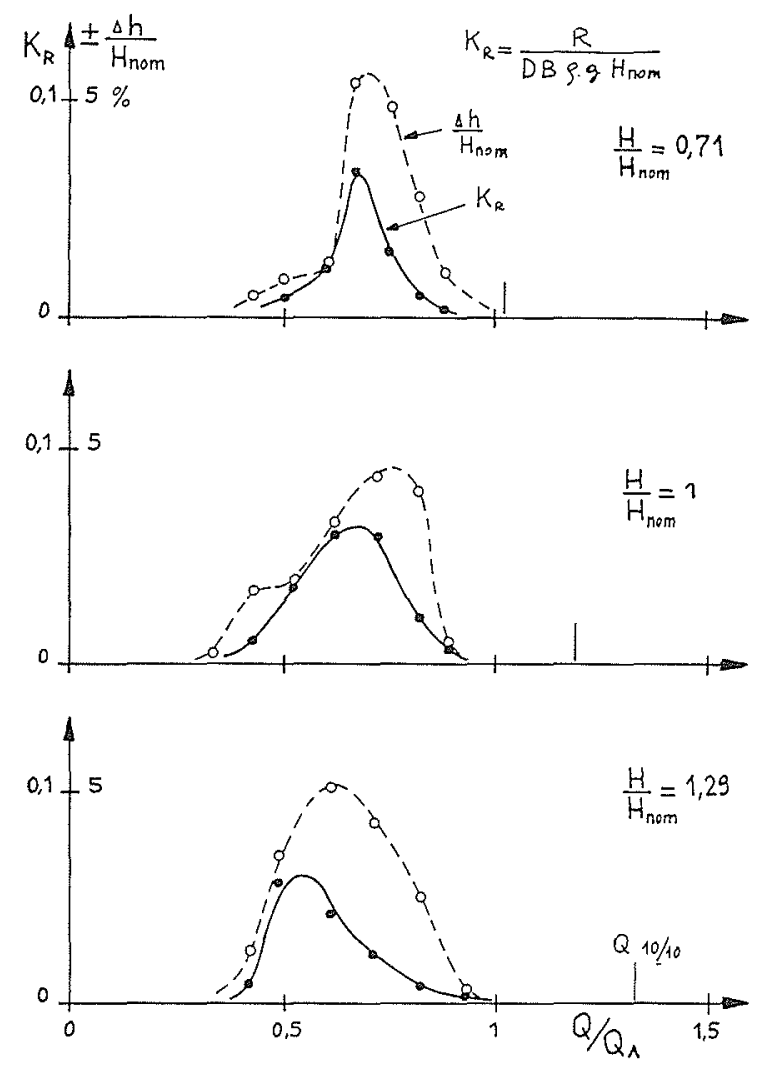

Figure $2-$ Turbine Francis $n_{q^{-}}=95$ : poussée radiale de torche et pulsation de pression sous roue en fonction du débit relatif.

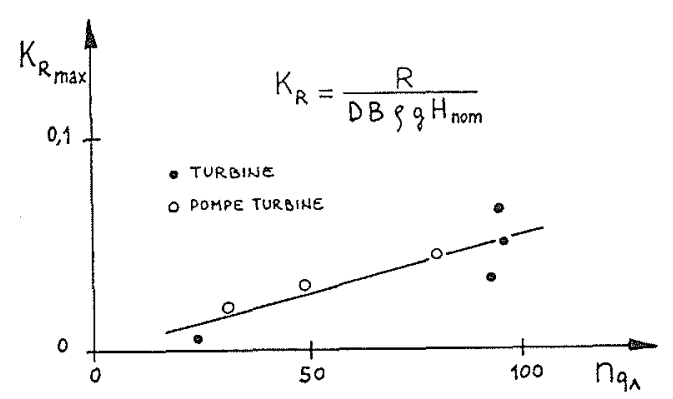

Figure 3 - Fonctionnement à débit partiel : poussée maximale de torche pour $H_{\text {nom }}$ en fonction de la vitesse spécifique.

Le diagramme de la figure 4 montre la poussée de torche mesurée à l'emballement, en fonction de l'ouverture du distributeur, sur le même modèle que des diagrammes précédents figures 1 et 2 .

La fréquence de rotation de la poussée est sensiblement le quart de la fréquence de rotation de la machine, tandis que l'ouverture du distributeur varie d'une façon assez semblable à celle de la figure 1 , c'est-à-dire qu'elle atteint son maximum pour une ouverture partielle du distributeur.

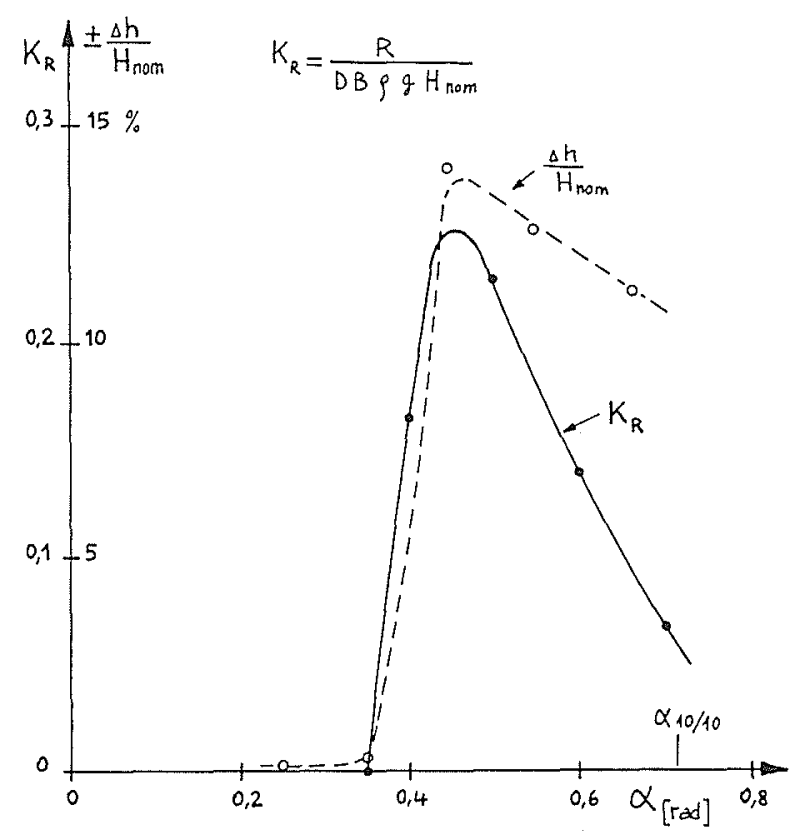

Figure 4 - Turbine Francis $n_{q-}=95$ : poussée radiale de torche et pulsation de pression sous roue à l'emballement.

Le diagramme de la figure 5 montre l'allure des coefficients de poussée radiale maximale de torche à l'emballement en fonction de la vitesse spécifique et pour différents modèles de turbine Francis et de pompeturbine.

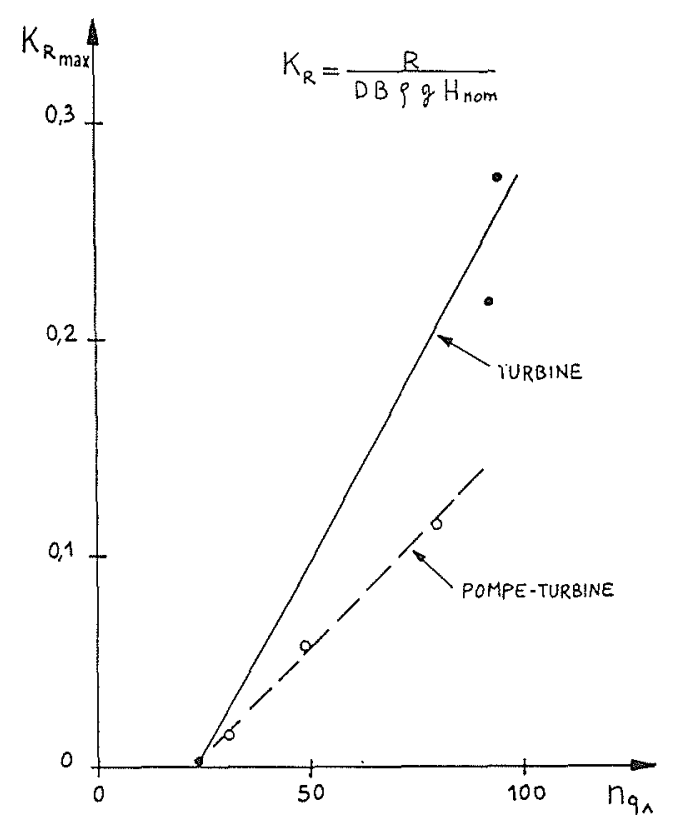

Figure 5 - Fonctionnement à l'emballement : poussée maximale de torche en fonction de la vitesse spécifique. 


\section{Poussée radiale aléatoire}

Contrairement à la poussée radiale de torche, qui a seulement lieu dans des conditions particulières de fonctionnement, la poussée radiale aléatoire, d'une façon plus ou moins sensible, peut être considérée comme présente dans tout le domaine de fonctionnement d'une machine hydraulique (cette donnée est caractérisée par une force qui varie dans le temps d'une façon aléatoire en grandeur et en direction).

L'interprétation des résultats d'essai sur modèle en termes rigoureux est très complexe, surtout quand il s'agit de fréquences élevées - égales à plusieurs fois celles de rotation - c'est-à-dire les fréquences qui s'approchent des fréquences flexionnelles propres de l'arbre du banc d'essai.

Sur le plan pratique, il semble opportum d'exprimer approximativement la grandeur de la poussée en question par les valeurs maximales de crête des fluctuations enregistrées en fonction du temps.

La poussée, ainsi mesurée, comprend évidemment toutes les composantes, y compris celles à basse fréquence et donc celle à la fréquence de la torche, lorsque celle-ci est présente.

Le diagramme 6 montre l'allure de la poussée aléatoire en fonction du débit pour une pompe turbine à vitesse spécifique moyenne fonctionnant en pompe à vitesse constante.

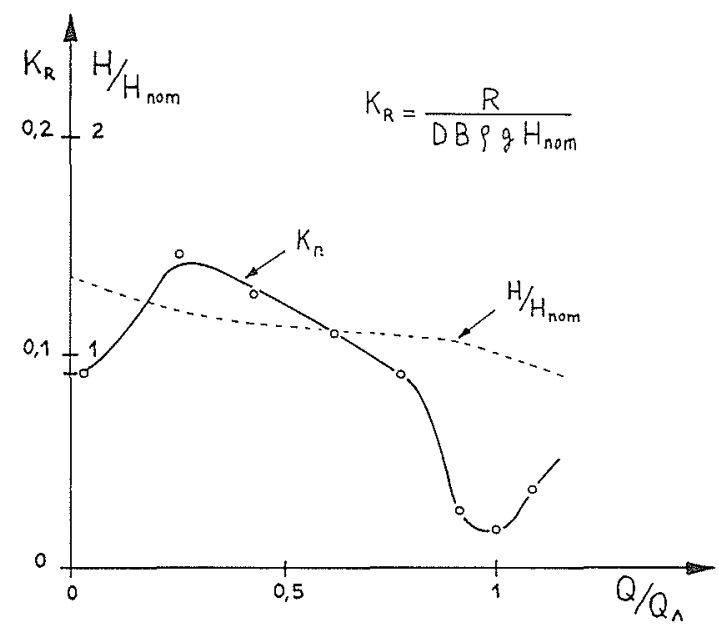

Figure 6 - Pompe-turbine $n_{q_{-}}=45$ : poussée aléatoire (crête) en fonction du débit pour un fonctionnement en pompe.

Le diagramme 7 montre - pour la même machine réversible - le domaine complet de la poussée aléatoire sous chute constante.

L'on peut remarquer que :

- dans le fonctionnement en pompe à débit réduit, la poussée aléatoire augmente fortement lorsque le débit diminue pour atteindre un maximum, puis redescendre pour atteindre une valeur encore remarquable pour $Q=0$;

- dans le fonctionnement en frein, l'on peut atteindre des valeurs plus élevées que celle susnommée ;

- dans les fonctionnements en suremballement, on atteint les valeurs de poussée maximales;

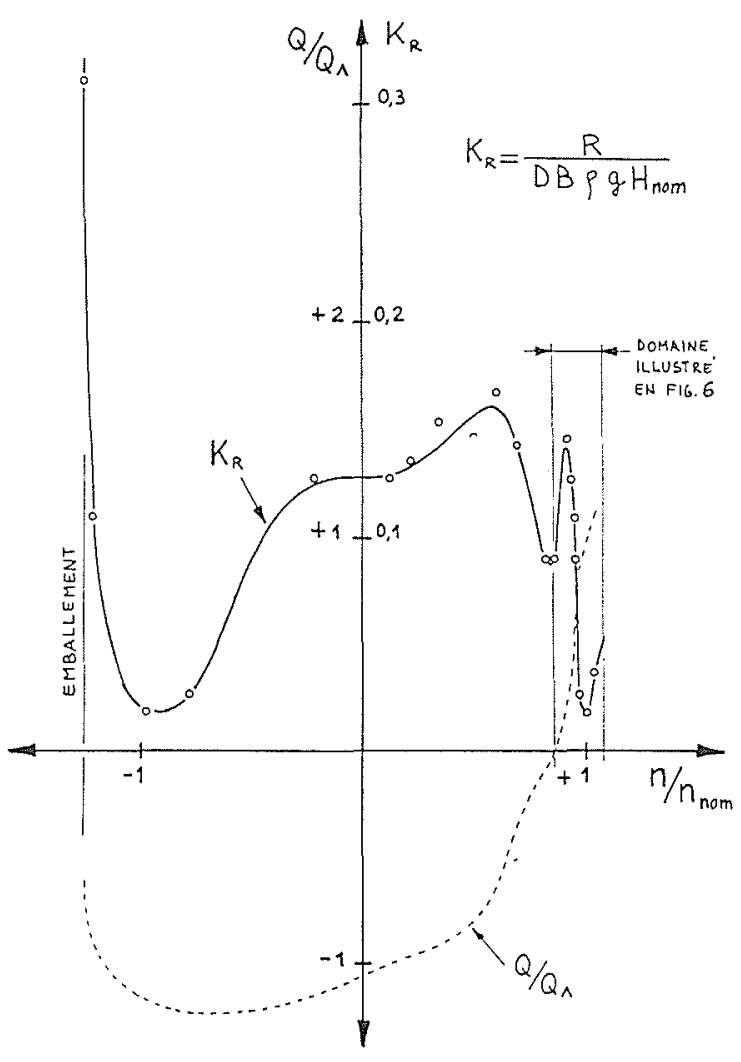

Figure 7 - Pompe-turbine $n_{q^{-}}=45:$ diagramme général de la poussée radiale aléatoire (crete) en fonction de la vitesse de rotation relative.

- les valeurs minimales de la poussée aléatoire se vérifient en coïncidence avec les domaines de fonctionnement normal de la machine, soit en pompe, soit en turbine.

\section{Conclusions}

En prenant en considération les machines hydrauliques pour les centrales hydroélectriques on peut arriver aux conclusions suivantes:

a) la poussée radiale fixe ne pose pas de problème technique ;

b) la poussée radiale tournant à la fréquence de torche ne pose également pas de problème particulier ;

c) la poussée radiale tournant à la fréquence de torche se vérifie à l'emballement et doit être prise en considération lors du projet mécanique de la machine, surtout s'il s'agit de turbines Francis à haute vitesse spécifique ; fique ;

d) la poussée aléatoire qui se manifeste dans les domaines de fonctionnement, loin du fonctionnement normal, présente des valeurs élevées qui peuvent conditionner le dimensionnement de la machine.

Sur ce sujet, toutefois, la discussion est ouverte, soit en ce qui concerne la précision de la mesure, soit en ce qui concerne l'interprétation des résultats. 


\section{Notations utilisées}

$f_{n} \quad=$ Fréquence de rotation de la machine

$f_{r} \quad=$ Fréquence de rotation de la torche

$R \quad=$ Poussée radiale (valeur de crête dans le cas aléatoire)

$R_{\chi}, R_{y}=$ Composantes de la poussée radiale dans les deux plans orthogonaux de l'arbre

$K_{r} \quad=$ Coefficient de poussée radiale

$H_{\text {nom }} \quad=$ Chute nominale de la machine

$\Delta h \quad=$ Amplitude de la pulsation de pression sous roue
Q. $\quad$ = Débit de rendement maximum (en pompe dans le cas d'une pompe-turbine)

$n_{q^{\wedge}} \quad=$ Vitesse caractéristique $=\frac{n \sqrt{Q}}{4 \sqrt{H_{\text {nom }}^{3}}}$

$D \quad=$ Diamètre de la roue maximum entrée turbine/sortie pompe

$=$ Hauteur du distributeur

$=$ Ouverture du distributeur

$=$ Densité de l'eau

$=$ Accelération de gravité.

\section{Discussions}

M. ZANETTI fait son exposé en projetant la figure 8 qui s'ajoute à celles du texte et qui montre, d'une façon schématique, la classification des types de poussées proposée.

Il le complète en se référant à l'intervention de $M$. BORCIANI à propos de l'origine du phénomène d'instabilité dans les turbines Francis à débit partiel et à la corrélation entre les différentes grandeurs pulsantes. Il montre la position de la poussée radiale dans ce contexte. On sait que les phénomènes qui sont toujours présents dans le fonctionnement à débit partiel, surtout lorsqu'il s'agit d'une turbine Francis à haute vitesse spécifique sont : la rotation de la masse d'eau sous roue et la rotation conséquente du champ de pression dans la même zone, ce qui donne naissance à la fluctuation dont il est question dans le mémoire Henry-Wegner-Graeser.

Ces phénomènes se produisent indépendamment de la valeur du sigma et - il faut considérer ce fait attentivement - indépen- damment de la forme du tuyau d'aspiration, soit-il courbe ou droit. Evidemment la poussée radiale tournant à la fréquence de la torche est liée aux deux phénomènes susmentionnés.

Voilà quelques résultats des essais en laboratoire effectués sur un modèle de turbine Francis à haute vitesse spécifique $\left(n_{q}=95\right)$. Le diagramme figure 9 montre l'évolution de quelques unes des grandeurs considérées en fonction de l'ouverture du distributeur lorsque la turbine fonctionne à débit partiel : poussée radiale, pulsation de pression sous roue, pulsation de chute et pulsation de débit. Toutes ces pulsations sont rapportées à la fréquence de torche.

On peut noter deux séries de courbes, celle à trait continu et celle en pointillé. La première concerne la solution classique d'une turbine Francis (avec coude d'aspiration), la deuxième se réfère à une solution expérimentale avec tuyau d'aspiration droit.

\begin{tabular}{|c|c|c|c|c|}
\hline TYPE DE POUSSEE & $\begin{array}{l}\text { SIGNAL SUR LA } \\
\text { PARTIE TOURNANTE } \\
\text { EN FONCTION } \\
\text { DU TEMPS } \\
\end{array}$ & $\begin{array}{l}\text { SPECTRE DU } \\
\text { SIENAL SUR LA } \\
\text { PARTIE TOURNANTE }\end{array}$ & $\begin{array}{c}\text { ORIGINE } \\
\text { PRESUMABLE }\end{array}$ & $\begin{array}{c}\text { CONATIONS } \\
\text { DE } \\
\text { FonCTIOAMEM. }\end{array}$ \\
\hline $\begin{array}{l}\text { FIXE PAR RAPPORT } \\
\text { AU STATOR }\end{array}$ & & & 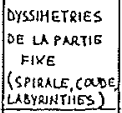 & roures \\
\hline 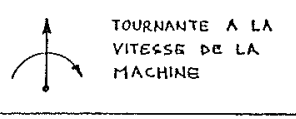 & t & $f$ & $\begin{array}{l}\text { DYSSIMETRES } \\
\text { DE LA PARTIE } \\
\text { TOURAAGTE } \\
\text { (CAMAU ROUE, } \\
\text { LAMRINTIES) }\end{array}$ & TOUTES \\
\hline $\begin{array}{l}\text { f. TOURNANTE A VITESSE } \\
\text { REDUTE (ENV. } 1 / 3 \\
\text { DE CELE DE LA } \\
\text { MACHINE) }\end{array}$ & -1 & $\mathrm{f}$ & TORCHE & $\begin{array}{l}\text { TURBINE } \\
\text { OERTTEL } \\
\text { PARTIEL } \\
\text { EMBRLLEHEKT }\end{array}$ \\
\hline$\ldots \quad$ aleatorre & $b i=t$ & & $\begin{array}{l}\text { BETACHEMENT } \\
\text { DE VENE, } \\
\text { rORBLLLONS, } \\
\text { ETE. }\end{array}$ & $\begin{array}{l}\text { LoIN DE } \\
\text { CELLES } \\
\text { MORMALES }\end{array}$ \\
\hline & 1 Tove & $\begin{array}{l}\text { FREQUENCE } \\
\text { FotAE } \\
\text { ROTIION }\end{array}$ & & \\
\hline
\end{tabular}

Figure 8 - Classification des types de poussées radiales. 

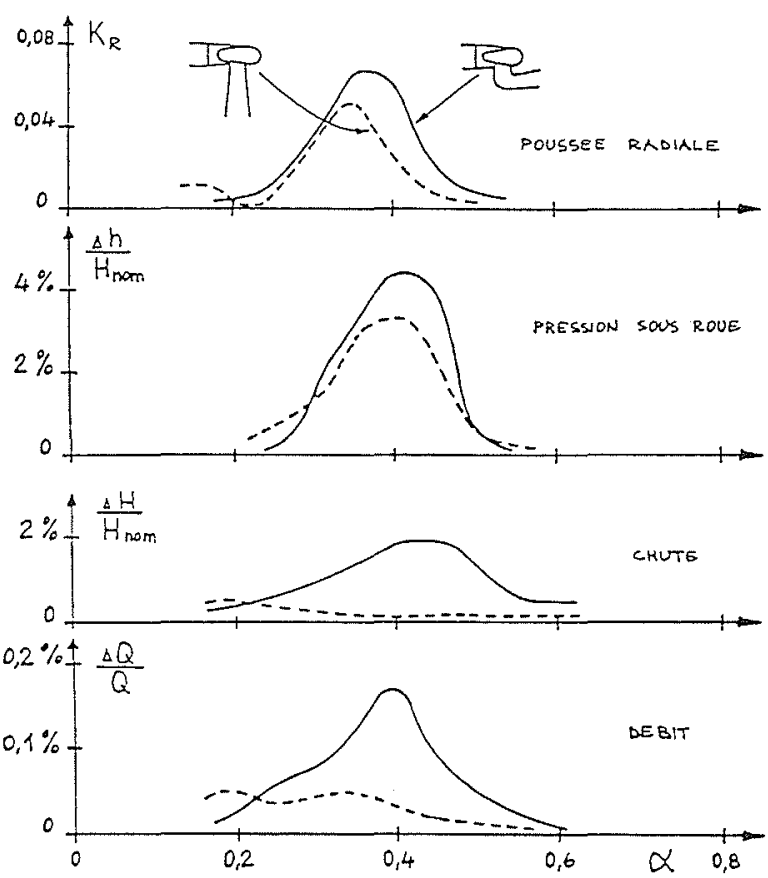

Figure 9 - Francis $n_{q^{\wedge}}=95$ : à débit partiel : comparaison entre poussée radiale et pulsations des autres grandeurs à la fréquence de la torche en fonction de l'ouverture du distributeur.

On voit que la poussée radiale, à la fréquence de la torche, a un comportement analogue aux fluctuations de pression sous roue, c'est-à-dire très peu influencé par la forme du tuyau d'aspiration, alors que là chute et le débit sont extrêmement sensibles à la forme du tuyau d'aspiration.

M. ROSSI, qui a participé à la réalisation du dispositif de mesure et qui l'utilise à présent, va le décrire en exposant l'histoire de son évolution et en complètant l'exposé de $M$. ZANETTI avec la projection de dispositives.

Le premier système que nous avons réalisé, il y a 5 ans environ est représenté par la figure $10 \mathrm{a}$.

Il était basé, comme on peut le voir, sur l'emploi de transducteurs électroniques qui relevaient les efforts transmis par la bague extérieure du palier guide à la partie statorique, selon deux axes orthogonaux.

Le système n'a pas donné de résultats satisfaisants, ni du point de vue de la répétibilité, ni de celui de la précision, malgré quelques efforts de perfectionnement. Le défaut intrinsèque était évidemment dû à la complexité du système hyperstatique.

Nous avons alors pensé qu'il valait la peine, dans le but de simplifier - au moins théoriquement - la fonction de trans-

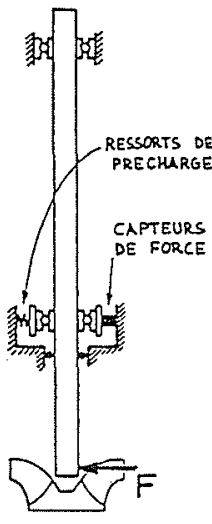

a

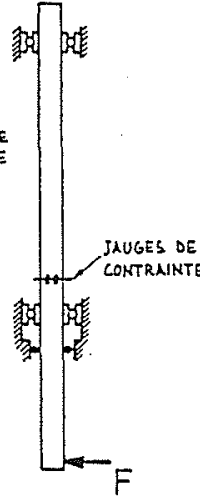

$b$

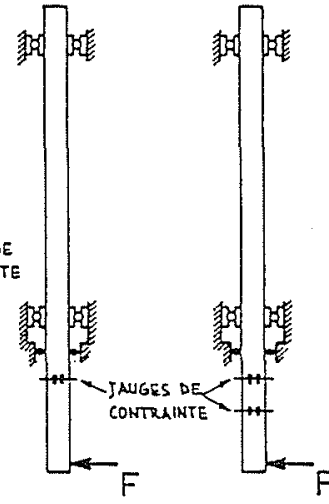

$\alpha$
Figure 10 - Expériences faites, sur le système de mesure de la poussée radiale. duction entre force à mesurer et signal de l'élément sensible, soit de réduire à une forme géométrique élémentaire la partie mécanique concernée par la mesure, soit de rapprocher le dispositif de mesure à la souce de la poussée radiale.

On a ainsi réalisé en 1976 la solution montrée par la figure $10 \mathrm{~b}$ qui utilise, en tant qu'éléments sensibles, des jauges de contrainte appliquées directement à une section transversale de l'arbre.

La transmission des signaux de la partie tournante à la partie fixe est effectuée au moyen de bagues tournantes et de brosses.

Ce système, malgré quelques imperfections qu'on mentionnera bientôt, s'est montré tout à fait valable et il nous a permis de parvenir à de bons résultats. On peut remarquer que la section d'applications des jauges de contrainte se trouve entre les deux paliers guides de l'arbre. Cette solution, à laquelle on a été amené pour raisons de sûreté liées aux problèmes d'isolation électrique, n'est pas tout à fait rationnelle, car la section mentionnée peut être soumise à des moments de flexion pas nécessairement produits par la force à mesurer.

En effet, au début on avait surestimé, dans certains cas, la valeur de la poussée radiale fixe, en raison de moments parasites transmis par le joint d'accouplement.

Un troisième système choisi récemment à la suite de l'expérience positive faite à propos de l'isolation des jauges de contrainte dans l'eau, est en phase de réalisation et est illustré dans la figure $10 \mathrm{c}$.

Il diffère du précédent par la position des jauges de contrainte qui sont situées à l'extérieur des paliers.

Les systèmes de figures $10 \mathrm{~b}$ et $10 \mathrm{c}$. permettent de résoudre les problèmes de mesure de façon satisfaisante du point de vue technique/pratique : mais ils sont encore insuffisants pour l'étude théorique de la poussée qui ne peut être développée d'une manière adéquate que par la détermination séparée des forces et des moments. C'est pour cette raison que nous avons construit, au cours de cette année, deux supports spéciaux, équipés de jauges de contrainte placées dans deux sections transversales différentes de l'arbre, comme le montre la figure $10 \mathrm{~d}$.

M. ROSSI termine son exposé en projetant des diapositives montrant les détails de l'application des jauges de contrainte, du système des bagues tournantes et des brosses fixes pour transmettre les signaux de la partie tournante à la partie fixe et l'équipement utilisé pour les calibrations.

M. le Président. - Je remercie M. ZANETTI et M. ROSSI de leur exposé.

Je pense que M. LIESS, de la Maison VOITH, voudrait présenter quelques observations sur des méthodes de mesures utilisées chez VOITH.

M. LIESS. - A la fin de son exposé, M. ZANETTI indique qu'il est nécessaire de considérer la fonction de transfert du modèle, si les fréquences de la poussée radiale sont proches des fréquences propres du modèle. C'est justement cette idée que nous avons essayé de réaliser en développant notre dispositif pour la mesure de la poussée radiale. En regardant, d'une manière générale, le problème consistant à mesurer la poussée radiale dynamique, on constate que d'une part, on veut connaître la poussée hydraulique mais que d'autre part, on ne peut mesurer que la réaction du banc d'essais à la poussée radiale.

Pour l'interprétation des résultats de mesure, il est indispensable de connaître la position relative des fréquences de la poussée radiale et des fréquences propres du modèle. La figure 11 montre schématiquement deux cas extrêmes :

a) Les fréquences propres du modèle sont hors du régime des fréquences intéressantes de la poussée radiale. L'évaluation des mesures se réduit alors à une multiplication simple par un facteur constant donné par l'étalonnage du système.

b) Les fréquences propres du modèle sont situées dans le domaine de fréquence de la poussée radiale. C'est le cas qui était mentionné par M. ZANETTI. L'évaluation des mesures doit alors être effectuée en utilisant la fonction de transfert du modèle.

D'après nos expériences, la gamme de fréquences des poussées radiales s'étend jusqu'aux environs de $200 \mathrm{~Hz}$. Si on veut réaliser un modèle dont la première fréquence propre soit bien au-delà de $200 \mathrm{~Hz}$, la structure, doit être extrêmement raide. Ceci pose alors des problèmes de mesure très sérieux, car le seul moyen pratique pour déterminer la réaction du modèle aux 
poussées hydrauliques est la mesure des déformations de certains éléments du modèle. La condition primordiale pour obtenir un signal de mesure convenable, conduit à un modèle dont la première fréquence propre relative aux mouvements radiaux de la roue est de l'ordre de $100 \mathrm{~Hz}$ au maximum (la roue étant dans l'eau).

Ceci indique que le cas $A$ de la figure 11 n'est pas réalisable et que l'on est obligé d'évaluer les mesures dynamiques en utilisant la fonction de transfert du modèle.

Pour rendre cette évaluation la plus facile possible, il est recommander de construire le modèle de façon telle qu'on obtienne une fonction de transfert simple dans la gamme des fréquences intéressantes.
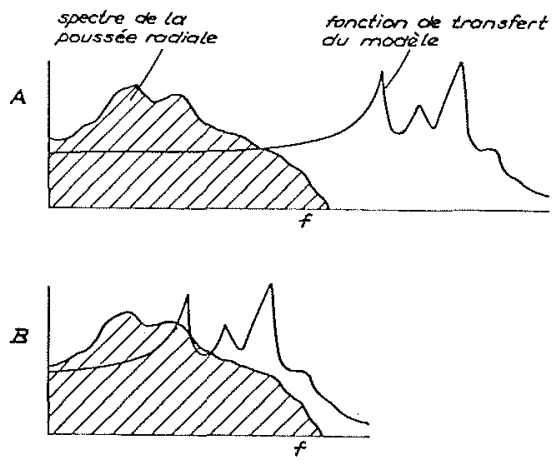

Figure 11 - Relations possibles entre les fréquences de la poussée radiale et les fréquences propres du modèle.

Or, on a essayé de réaliser un système oscillateur simple, dont les équations de mouvement peuvent être résolues directement. La figure 12 montre le schéma de l'oscillateur, composé d'une masse $M$, d'un ressort $K$ et d'un amortisseur $C$, et donne les équations de mouvement et la fonction de transfert. La force exitatrice $F_{R y}$ correspond à la poussée radiale hydraulique et la force de base $F_{L y}$ à la force de réaction mesurée.

Le palier de mesure construit pour la détermination des poussées radiales dynamiques est représenté schématiquement par la figure 13. Les parties essentielles sont l'arbre, les roulements et un anneau de mesure equipé de jauges de contrainte de façon telle qu'on puisse mesurer les composantes des forces dans deux directions perpendiculaires $x$ et $y$ (Fig. 5). La relation entre la poussée radiale et le signal électrique des jauges de contrainte est linéaire. L'anneau de mesure n'est pas sensible aux poussées axiales agissant sur la roue. L'accouplement entre l'arbre du palier et la machine électrique ne peut transmettre que le couple de rotation, mais pas de forces radiales axiales.

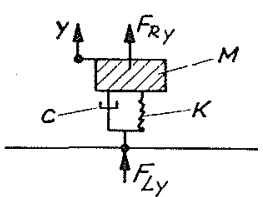

$$
\begin{aligned}
& \text { Système } d^{\prime} \text { un oscillateur simple } \\
& \begin{array}{c}
M \frac{d y}{d t^{2}}+c \frac{d y}{d t}+K_{y}=F_{R_{y}} \\
c \frac{d_{y}}{d t}+K_{y}=F_{L y}
\end{array}
\end{aligned}
$$

Equations de mouvement

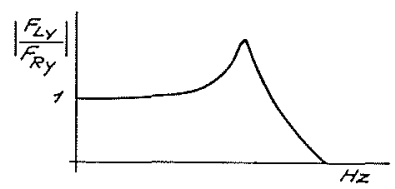

Figure 12 - Fonction de transfert d'un oscillateur simple.

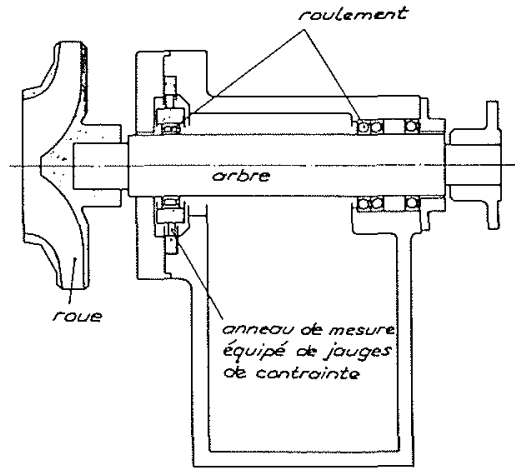

Figure 13 - Palier de mesure pour la poussée radiale.

La fonction de transfert du palier correspond bien à la courbe théorique de l'oscillateur simple comme l'indique la figure 14 sur laquelle sont représentées les courbes mesurées avec la roue dans l'air et dans l'eau. Pour la roue dans l'eau, il est nécessaire de filtrer le signal de mesure par un filtre passe-bas avec une fréquence limite de $200 \mathrm{~Hz}$. Les facteurs constants $M, C, K$ des équations de mouvement sont déterminés à partir des fonctions de transfert mesurées avec le modèle complètement assemblé et rempli d'eau.

L'évaluation des deux signaux de mesures est faite de la manière suivante :

- les signaux analogiques sont convertis en séries de valeurs digitales qui sont introduites dans un ordinateur ;

- le filtrage passe-bas est exécuté ensuite numériquement. L'utilisation d'un filtre numérique a l'avantage de permettre de choisir la fonction de transmission du filtre librement et sans déplacement des fréquences ;

- à partir des signaux filtrés, qui correspondent aux forces de base du simple oscillateur (Fig. 12), les composantes de la poussée radiale $F_{R x}$ et $F_{R y}$ sont calculées à l'aide des équations de mouvement;

- l'addition vectorielle de ces composantes fournit finalement la grandeur et la direction de la poussée radiale.
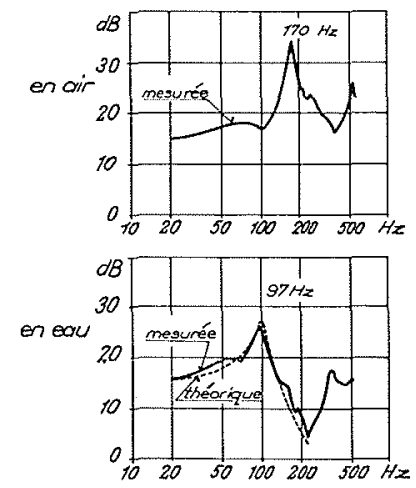

Figure 14 - Fonction de transfert du palier de mesure.

La figure 15 montre la suite des courbes obtenues pour la détermination de la poussée radiale dans le cas d'une pompeturbine avec vanne fermée.

Pour déterminer les sollicitations de l'arbre et des paliers de la machine industrielle, les composantes de la poussée radiale sont transférées du modèle au prototype et introduites comme forces excitatrices dans le calcul du comportement vibratoire de la ligne d'arbre. La comparaison des prévisions avec les mesures effectuées sur la machine industrielle pendant la mise en marche montre une très bonne correspondance.

M. le Président. - Je vous remercie beaucoup pour votre contribution très intéressante. 


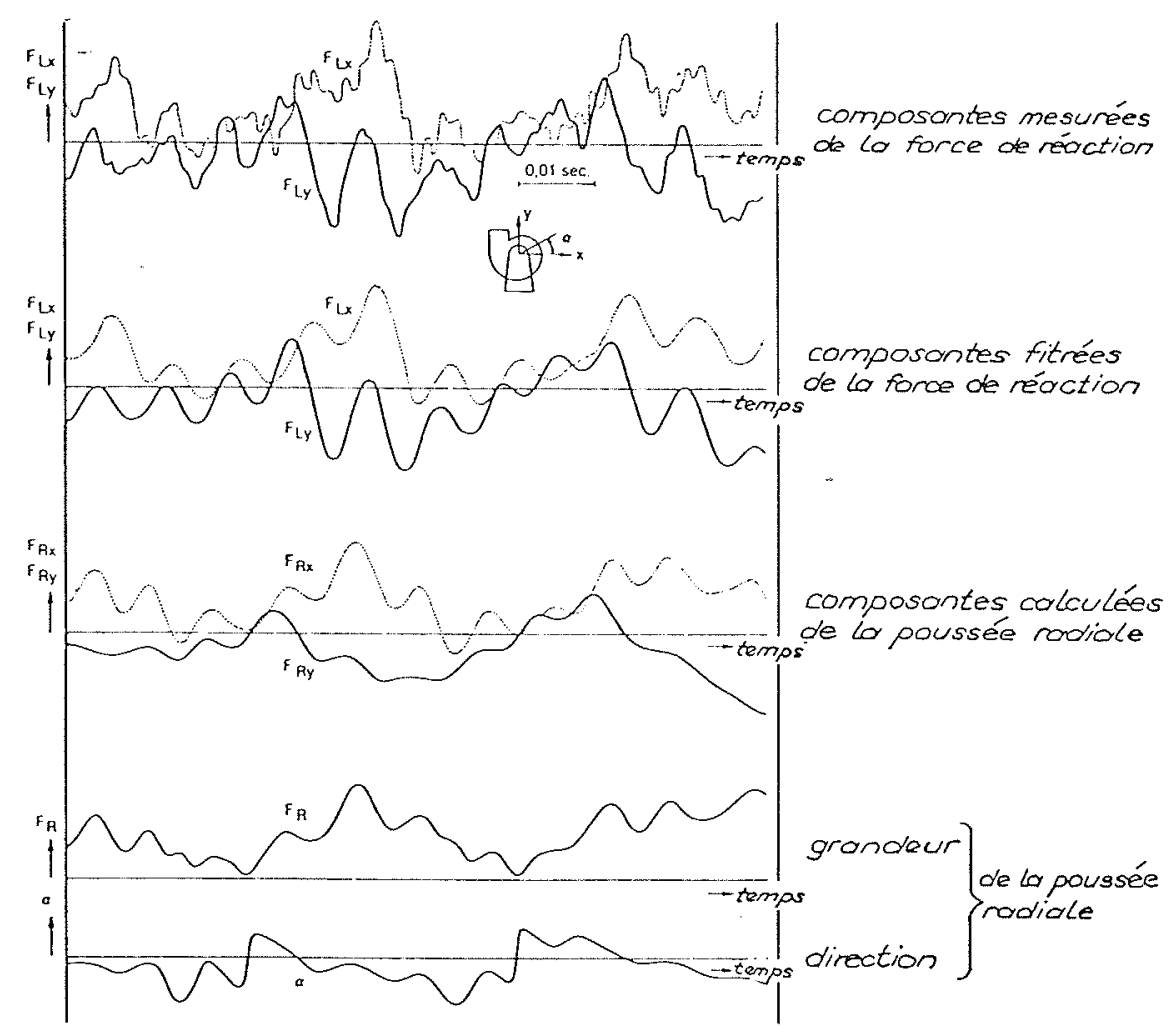

Figure 15 - Détermination de la poussée radiale : pompage à vanne fermée d'une pompe-turbine.

M. BOR CIANI. - Je pense qu'il faut savoir s'il est préférable d'effectuer la mesure sur la partie tournante ou sur la partie statique. Nous avons commencé les mesures sur la partie statique et nous sommes ensuite passés à la partie tournante.

M. LIESS a développé un système qui prévoit la mesure sur la partie fixe.

Il serait intéressant de comparer les résultats obtenus par les deux systèmes afin d'examiner lequel est le plus simple et le plus fiable.

Au dessous de la fréquence de $80 \mathrm{~Hz}$, le système que nous utilisons ne nécessite pas le passage à travers la fonction de transfert, qui d'autre part, devrait être plus simple.

Je voudrais savoir s'il suffit d'atteindre la fréquence de $80 \mathrm{~Hz}$ et s'il y a quelques raisons techniques - suivant vos opinions et expériences - en faveur du système adopté par nous.

M. LIESS. - La raison pour laquelle nous avons adopté le système de mesure sur la partie fixe est que l'on veut aussi considérer des fréquences supérieures, par exemple au $50 \mathrm{~Hz}$. Et pour cela, il faut tenir compte de la fonction de transfert.

M. le Président. - En fait, je dirais que les deux systèmes sont équivalents. Vous pouvez très bien réfléchir sur la fonction de transfert avec votre système et voir s'il $\mathrm{y}$ a une influence sur vos mesures, comme l'a fait la Maison VOITH sur la partie statique. Il suffirait, par exemple, de mettre sur l'arbre un balourd et de faire tourner ce balourd à très grande vitesse, puis de voir quelle est la réponse de vos jauges de contraintes pour savoir si dynamiquement, vous avez des mesures correctes ou non.

D'ailleurs, on pourrait aussi faire une analyse des réponses par la même fonction de transfert aléatoire, et même des mesures aléatoires sur les fonctions de transfert. Je ne sais pas si, du point de vue pratique, il est plus simple d'adopter l'un ou l'autre système ; l'intérêt est précisément d'avoir les deux. Vous donnez les résultats sur le système VOITH et le système HYDROART et on les compare, mais les deux systèmes sont équivalents, à l'étude de la fonction de transfert près, dirais-je.

M. ZANETTI. - Je suis d'accord. Mais il est plus simple de mesurer la fonction de transfert de la partie toumante ou de la calculer. Il est plus difficile de schématiser les liaisons hyperstatiques dans le cas du calcul de la fonction de transfert de la partie fixe.

M. LIESS. - Je crois que l'essentiel de notre question est de savoir s'il est nécessaire de faire cet eftort ou non. Pour y répondre il faudrait analyser le résultat de nos dépouillements en fréquences pour voir si, vraiment, les forces hydrauliques vont jusqu'à $200 \mathrm{~Hz}$.

$\mathrm{Si}$ on peut se contenter de fréquences inférieures à $50 \mathrm{~Hz}$ les deux systèmes sont équivalents. Mais il faudrait tout d'abord faire cette analyse.

M. le Président. - En fait, cette étude peut être faite pour un système ou pour un autre. Il suffit de trouver la fonction de transfert, c'est-à-dire la réponse du système type ZANETTI et la réponse du système type LIESS, et on devrait arriver au même résultat, sauf que c'est encore plus compliqué parce qu'il peut y avoir un mélange des deux. Mais les fonctions de transfert peuvent être encore beaucoup plus complexes que ce qu'on imagine.

M. JAQUET. - Je voudrais dire quelques mots sur la mesure de la poussée radiale. Nous avons appliqué les deux systèmes et, depuis quelques années, nous employons également le système sur l'arbre rotatif, c'est-à-dire que nous avons un arbre avec les deux paliers, comme cela a été montré par HYDROART, et, sur cet arbre, nous avons disposé deux sections munies de jauges de contraintes (Fig. 16) avec lesquelles on mesure la poussée radiale sur la roue.

Maintenant, ce que l'on mesure dans ces deux sections munies de jauges de contrainte, ce n'est pas une force, mais un couple, c'est-à-dire cette force multipliée par le bras de levier. Cette force peut se trouver à un endroit quelconque dans le plan de la roue et il intervient. C'est cette mesure double qui est également adoptée par HYDROART. Il y a, entre les deux sections, une longueur qui est connue et on mesure deux signaux : un dans cette section, l'autre dans la seconde.

Il y a deux équations: on mesure un couple $M 1$ et un couple $M 2 ; M 1$ équivaut à $(a+b) F_{r}$ et $M 2$ équivaut à $b . F_{r}$. Ces deux équations permettent ensuite de déterminer la grandeur de la poussée radiale et sa position par rapport à la roue. 


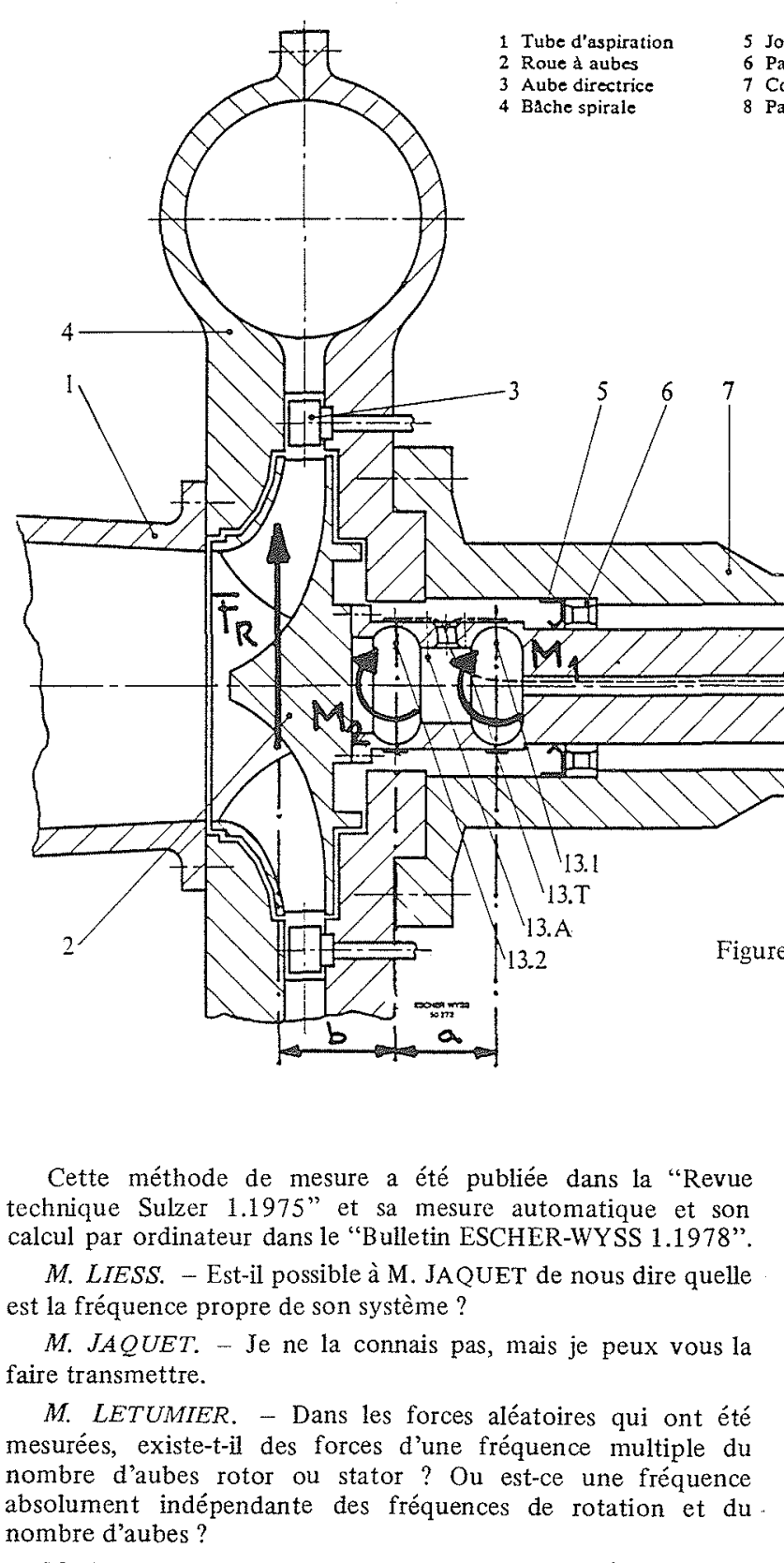

M. ZANETTI. - On trouve quelquefois une fréquence qui

cette Sethode de me" a ce publiee dans la "Revue calcul par ordinateur dans le "Bulletin ESCHER-WYSS 1.1978".

M. LIESS. - Est-il possible à M. JAQUET de nous dire quelle est la fréquence propre de son système?

M. JAQUET. - Je ne la connais pas, mais je peux vous la transmettre. nombre d'aubes rotor ou stator ? Ou est-ce une fréquence absolument indépendante des fréquences de rotation et du NETI. On trouve quelquefois une frequence qui $\begin{array}{lll}5 \text { Joint d'arbre } & 9 \text { Conduit des cables } & 13.1 \text { Plan de mesure 1 } \\ 6 \text { Palier radial } & 10 \text { Porte-balaiz } & 13 . T \text { Plan de mesure T } \\ 7 \text { Corps de palier } & 11 \text { 24 bagues de contact } & 13 . \text { Alan de mesure A } \\ 8 \text { Palier radial et de butee } & 12 \text { Accouplement a dents } & 13.2 \text { Plan de mesure 2 }\end{array}$ correspond au nombre des aubes de la roue multipliée par la fréquence de rotation. Mais la valeur n'est pas jugée très importante; c'est faible par rapport aux autres forces.

$M$. le Président. - Je voulais ajouter un point. Il y a non seulement un effort qui peut varier, mais il y a aussi un couple pur qui peut être appliqué à la roue et qui peut se mesurer avec ces ponts.

On a trouvé, non pas sur des machines Francis, mais sur des Kaplan et sur des Bulbes, des couples purs qui étaient appliqués à la roue.

M. SCHIAVELLO. - Est-ce qu'entre les sections où on a mesuré $M 1$ et $M 2$ et la section de la roue, il n'y a pas de paliers lisses ?

M. JAQUET. - Il n'y a rien du tout. 


\section{Abstract}

Radial thrust in hydraulic machines, laboratory testing

The radial thrust exerted by the flow on the runner plays an important role among the loads acting on a hydraulic machine at the different operating conditions.

It is useful to proceed to a classification, though schematic, of the various types of thrust which may occur.

A first type of thrust, the fixed one, is due to dissymmetries in the stationary parts. It just has the effect to bend the shaft line and to load the guide bearings.

A second type of thrust is the dynamic thrust which causes vibrations and has consequently more importance in machine designing, at least in the case of machines for hydroélectrical plants.

We shall distinguish the dynamic thrusts of the rotating type from those of the random type.

Among the former, the thrust rotating at the rope frequency, phenomenon characterising the operation of a Francis turbine at part load or at runaway conditions, is to be stressed. In extreme cases this thrust may cause the sliding of the rotating on the stationary part at the labyrinths.

The latter occul in far from normal operating conditions and are to be taken into account in particular when calculating fatigue strenght.

The paper includes a series of diagrams showing the results obtained from model tests of high specific speed Francis turbines and pump-turbines. They relate above all to the dynamic thrusts and can be summarised as follows:

a) Radial thrust rotating at the rope frequency: the phenomenon is directly associated with that of the pressure pulse below the runner and increases with the specific speed of the unit. Some diagrams show that quite a similar phenomenon occurs in runaway operation: the thrust attains its maximum value in high specific speed Francis turbines.

b) Random thrust: it occurs at different operating conditions, reaching in case of pump-turbines - its peak values at runaway, in the braking mode and in pump mode at strongly reduced flow. 